\title{
Modulation of fibronectin expression in the central nervous system of Lewis rats with experimental autoimmune encephalomyelitis
}

\section{M.C.A. De-Carvalho ${ }^{1}$, \\ L.M.C. Chimelli2 and \\ T. Quirico-Santos 3,4}

\author{
Departamentos de ${ }^{2}$ M orfologia, Universidade do Rio de Janeiro, 2Patologia, \\ Universidade Federal do Rio de Janeiro, ${ }^{3}$ Biologia Celular e Molecular, \\ Instituto de Biologia, Universidade Federal Fluminense, and ${ }^{4}$ Imunologia, \\ Instituto O swaldo Cruz, Rio de Janeiro, RJ, Brasil
}

\section{Correspondence \\ T. Q uirico-Santos \\ Laboratório de Patologia Celular \\ Departamento de Biologia Celular \\ e Molecular, IB, UFF \\ O uteiro de São João Batista, s/n \\ 24020-150 Niterói, RJ \\ Brasil \\ Fax: + 55-21-719-5934 \\ E-mail: tquirico@urbi.com.br \\ Presented at the 5th Brazilian Symposium on Extracellular Matrix - SIMEC, Angra dos Reis, \\ RJ, Brasil, September 7-10, 1998.}

Research supported by CAPES.

Received November 17, 1998 Accepted December 14, 1998

\section{Abstract}

Fibronectin (FN), a large family of plasma and extracellular matrix (ECM) glycoproteins, plays an important role in leukocyte migration. In normal central nervous system (CNS), a fine and delicate mesh of FN is virtually restricted to the basal membrane of cerebral blood vessels and to the glial limitans externa. Experimental autoimmune encephalomyelitis (EAE), an inflammatory CNS demyelinating disease, was induced in Lewis rats with a spinal cord homogenate. During the preclinical phase and the onset of the disease, marked immunolabelling was observed on the endothelial luminal surface and basal lamina of spinal cord and brainstem microvasculature. In the paralytic phase, a discrete labelling was evident in blood vessels of spinal cord and brainstem associated or not with an inflammatory infiltrate. Conversely, intense immunolabelling was present in cerebral and cerebellar blood vessels, which were still free from inflammatory cuffs. Shortly after clinical recovery minimal labelling was observed in a few blood vessels. Brainstem and spinal cord returned to normal, but numerous inflammatory foci and demyelination were still evident near the ventricle walls, in the cerebral cortex and in the cerebellum. Intense expression of $\mathrm{FN}$ in brain vessels ascending from the spinal cord towards the encephalon preceded the appearance of inflammatory cells but faded away after the establishment of the inflammatory cuff. These results indicate an important role for $\mathrm{FN}$ in the pathogenesis of CNS inflammatory demyelinating events occurring during EAE.

\section{Introduction}

Experimental autoimmune encephalomyelitis (EAE), a T cell-mediated disease, is considered to be the animal model $(1,2)$ of multiple sclerosis, a chronic inflammatory demyelinating disease of the human central

\section{Key words}

- Experimental autoimmune encephalomyelitis (EAE)

- Extracellular matrix

- Fibronectin

- Central nervous system 
tein (MAG), S-100ß and glial fibrillar acidic protein (GFAP) specific T-cells (3-5). Lewis rats usually develop a monophasic disease with onset about 9 to 12 days after immunization and spontaneous recovery occurs by day 20 , with animals becoming resistant to re-induction of the disease. Edema and demyelination are responsible for the development of neurological deficits which include paresis and paralysis of the tail and hind limbs (2).

The main histopathological features within the CNS of affected animals are widespread lesions characterized by perivenular cuffs and slight demyelination in close proximity to inflammatory areas (3). Alteration in the permeability of the blood-brain barrier (BBB) precedes the entrance into the CNS of macrophages and activated $T$ cells secreting various cytokines (6-8). Trafficking of specific leukocyte subsets is mediated by a multistep process that in many circumstances involves leukocyte rolling, adhesion to endothelial ligands through activated integrins and diapedesis (9). Among the earliest events implicated in the pathology of CNS inflammatory demyelinating diseases is an increased tendency for circulating leukocytes to cross the BBB (10). Various lines of evidence indicate an important role for extracellular matrix (ECM) receptors and ligands in regulating lymphocyte migration, function and positioning (11,12). Indeed, antibodies against $\alpha 4 \beta 1$ integrin (13), a receptor for both fibronectin (FN) and adhesion molecule (VCAM-1), prevents development of EAE.

The ECM of normal brain tissue (14) is mainly composed of aggregates and polymers of macromolecules, particularly proteoglycans, though with less abundant expression of basement membrane components (type IV collagen, laminins and FN). The basement membrane, besides acting as a barrier to macromolecules and cells, also provides a substratum for cell attachment, spreading, differentiation, migration and ap- optosis $(15,16)$. Interactions of individual ECM components with specific cell surface molecules (integrin receptors) and proteoglycans initiate a cascade of signal transduction leading to varied short-term or persistent cellular responses. FN is expressed in a very specific spatial and temporal manner, important both for neuronal migration and outgrowth during development. FN is also necessary for remodeling injuried brain tissue (15,17-19), promoting nerve regeneration and converting quiescent astrocytes to a proliferating phenotype.

There is an important relationship between microvascular permeability, FN deposits along with fibrin/fibrinogen and clinical course of EAE, rather than the cellular infiltrates which persist even after clinical recovery $(14,20,21)$. This study aimed to characterize subtle changes of FN expression in the CNS microenvironment that may be influencing early events in the pathogenesis and resolution of inflammation in Lewis rats developing active EAE.

\section{Material and Methods}

\section{Animals}

Lewis (LEW) rats were bred in the animal house of the Department of Cellular and Molecular Biology (Institute of Biology, Universidade Federal Fluminense). Female rats aged 6-8 weeks and weighing 100-150 g were kept in plastic cages containing pine chips, at constant temperature $\left(20^{\circ} \mathrm{C}\right)$ and with a light cycle of 12:12 h. Pelleted-enriched diet (Nuvital, PR, Brazil) supplemented once a week with vitamins and acidified water were provided ad libitum.

\section{Induction of active EAE}

Adult guinea pig spinal cord (GPSC) homogenized in a grinder with $0.95 \%$ saline was emulsified at a 1:2 ratio with incomplete Freund's adjuvant (IFA) (Sigma Chemical 
Co., St. Louis, MO, USA) containing $4 \mathrm{mg} /$ $\mathrm{ml}$ of Mycobacterium tuberculosis $\mathrm{H} 37 \mathrm{Ra}$ (Difco Laboratories, Detroit, MI, USA) to prepare complete Freund's adjuvant (CFA). Each rat was immunized once in the hind footpads with $100 \mu \mathrm{l}$ of this sensitizing emulsion (3). Three control groups were used: sex-age-paired normal LEW rats, LEW rats inoculated with $100 \mu$ saline-IFA plus $M$. tuberculosis, and LEW rats inoculated with $100 \mu \mathrm{l}$ GPSC-IFA emulsion lacking $M$. tuberculosis. Each group consisted of 6-8 animals. Each rat was weighed at the time of sensitization and just before sacrifice at the pre-clinical stage, 7 days post-immunization (7 d.p.i.), onset of disease (10 d.p.i), paralytic phase (14 d.p.i.), after clinical recovery (30 d.p.i) and after total recovery (4 months p.i).

\section{Clinical assessment of EAE}

Rats were observed daily for assessment of neurological signs. The severity of the disease was scored according to the following system: 0 - no evident neurological signs; 1 - limp tail; 2 - partial paralysis of the hind limbs, unsteady gait; 3 - moderate paraparesis, voluntary movements still possible; 4 paraplegia or tetraplegia, and 5 - moribund state.

\section{Processing of CNS tissues}

LEW rats were killed by cardiac perfusion under ether anesthesia. Animals were perfused through the left ventricle initially with a washing buffered saline solution containing $0.1 \%$ lidocaine and then with $10 \%$ buffered formalin. Encephalon and spinal cord were carefully removed from the skull and vertebral canal. Tissues were further maintained for 5 days in formalin-buffered fixative, $\mathrm{pH} 7.2$, for histochemical analysis, but only for $24 \mathrm{~h}$ for immunohistochemistry. For anatomopathological studies, we obtained four coronal sections of the encephalon anterior and posterior to the optic chi- asm, sagittal sections of the brainstem and cerebellum, and transverse and longitudinal sections of cervical, thoracic and lumbosacrococcygeal spinal cord segments. This material was then embedded in paraffin.

\section{Histological staining}

Five- $\mu \mathrm{m}$ sections of wax-embedded brain tissue and spinal cord fragments were stained with hematoxylin-eosin (HE) and 8- $\mu \mathrm{m} \mathrm{sec-}$ tions with luxol fast blue to assess demyelination.

\section{Immunolabelling}

Five- $\mu \mathrm{m}$ thick paraffin sections settled on silane (Sigma) pre-coated slides were dewaxed and rehydrated. Endogenous peroxidase activity was inhibited by incubation in three sequential baths of 20 min each with $3 \%$ hydrogen peroxide in methanol solution. Slides were then placed in plastic jars containing citrate buffer, $\mathrm{pH} 6.0$, and heated for three cycles of 3 min each at high power in a conventional microwave oven. Special attention was paid to checking inter-cycles in order to prevent tissue dryness.

Sections were sequentially incubated in a moist chamber for $1 \mathrm{~h}$ at room temperature with purified IgG goat serum (Sigma) diluted 1:100 followed by further overnight incubation at $4^{\circ} \mathrm{C}$ with Tris-buffered saline (TBS), pH 7.2, containing 3\% skimmed milk and $2.5 \%$ bovine serum albumin (BSA) type V (Sigma). Optimal concentration of monoclonal anti-FN antibody clone MAB 1940 (Pharmigen, San Diego, CA, USA) was applied to sections, followed by incubation at room temperature in a moist chamber for 60 min. A secondary peroxidase-conjugated goat anti-rabbit IgG $(\mathrm{H}+\mathrm{L})$ antibody (Zymed Laboratories Inc., South San Francisco, CA, USA) diluted 1:700 in TBS-BSA was added and incubated for $50 \mathrm{~min}$ at room temperature.

After thoroughly washing with PBS, en- 
zyme activity was developed with 3,3'-diaminobenzidine as peroxidase substrate in the presence of hydrogen peroxide. All sections were lightly counterstained with Mayer's hematoxylin, dehydrated and mounted in Canada balsam. Sections from every animal were examined.

\section{Results}

\section{Clinical course of EAE}

Disease onset in LEW rats immunized with GPSC-CFA generally occurred at 10 d.p.i., with affected rats presenting a limp tail (grade $=1)$. Special care was taken by providing water in a bottle with a longer sipper and pelleted enriched food in a container placed on the floor of the cage. There was no significant change in body weight among groups.

The clinical-neurological deficits of EAErats included progression into unsteady gait (grade $=2$ ) followed shortly thereafter (two days later) by moderate paraparesis and great difficulty in performing hind limb movements (grade $=3$ ). Later on, at 14 d.p.i affected rats presented total paralysis (grade $=4$ ) of the posterior portion of the trunk and hind limbs. Neither tetraplegia nor moribund state (grade $=5$ ) was observed in any affected EAE rat. Progression to death certainly might have occurred if disabled rats had not been treated. The recovery period (30 d.p.i.) initiated $24 \mathrm{~h}$ after paralysis, with a reduction of neurological deficits and subsequent clinical improvement, with rats running and climbing in the cage. Control groups did not present evident neurological sign of EAE, even those inoculated with GPSC homogenate.

Animals sacrificed during the pre-clinical stage (7 d.p.i.) did not present neurological signs. One group of EAE rats was accompanied through every phase until 4 months p.i. From the time of clinical recovery (30 d.p.i.) EAE rats did not show recurrence of clinical signs.

\section{Histopathological findings}

Hematoxylin-eosin sections of control rats immunized either with saline plus CFA, or GPSC plus IFA showed (Figure 1A) characteristic normal histology of nervous tissue at different levels of the spinal cord and encephalon. LEW rats immunized with GPSC plus CFA sacrificed in the pre-clinical phase (7 d.p.i.) of disease also showed normal CNS histology. During the onset of the disease (10 d.p.i.) LEW rats with grade 1 clinical evidence (limp tail) showed only minor changes in the histoarchitecture of the spinal cord. Rats with partial paralysis of the hind limbs (grade 2) showed mild leukocyte infiltration in the meninges and adjacent to the dorsal horn (Figure 1B), but not in the encephalon. The main CNS blood vessels affected in the disease were venules, with preservation of the capillary bed and meningeal great vessels.

In contrast, rats with full blown disease (14 d.p.i.) in the paralytic phase (grade 3 and grade 4 ) showed wide scattered perivenular mononuclear cell foci at different levels of spinal cord and brainstem (Figure 1C). Inflammatory cuffs were distributed in the meninges, in the white matter and also in the gray matter (Figure 1D). Only few inflammatory cuffs were observed in the thalamus and near third ventricle wall.

After clinical recovery (30 d.p.i.) affected rats showed few inflammatory foci in the meninges and parenchymal vessels at different levels of the spinal cord and brainstem. Nevertheless, sections of cerebellum, midbrain and forebrain showed numerous mononuclear cell infiltrates on the vessel walls and nervous parenchyma. Inflammatory foci in the circumventricular area were mainly localized in the proximity of the inferior and anterior horns of the lateral ventricles. Some inflammatory foci were also found in the cerebral cortex and cerebellar white matter. 

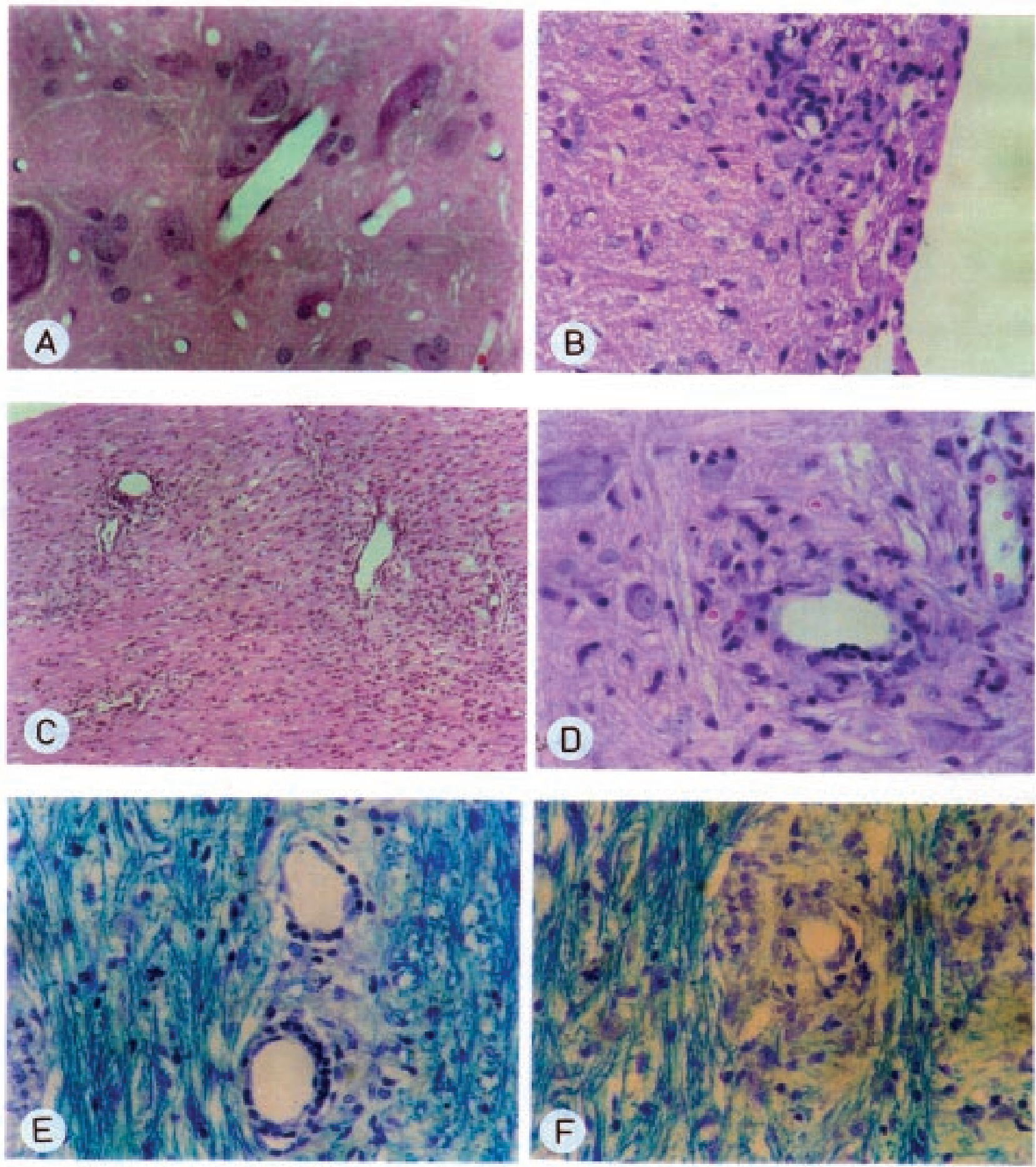

Figure 1 - Photomicrograph of wax-embedded CNS sections from a normal Lewis rat (A) and a rat with EAE (B-D) stained with hematoxylin-eosin. A, Normal cervical spinal cord gray matter microvasculature adjacent to neuropil, venules and capillaries lined with single endothelium. B, Transverse section of a cervical spinal cord segment from a LEW rat sacrificed at the onset (10 d.p.i.) of the disease, showing an inflammatory cuff near the dorsal horn. C, Longitudinal section of brainstem white matter from a rat with full blown disease (14 d.p.i.) showing scattered perivenular inflammatory cuffs. $D$, Section of a cenvical spinal segment from a rat in the paralytic phase (14 d.p.i.) showing a perivenular inflammatory infiltrate in the gray matter. E, $F$, CNS sections of lumbosacrococcigeal spinal cord segment from rats in the paralytic phase (grade $=4)$ stained with luxol fast blue showing $(E)$ a perivenular area of demyelination adjacent to inflammatory cuffs and (F) demyelinating plaque. Magnification: 400X (A,B,D,E,F) and 100X (C). 
Interestingly, EAE rats sacrificed 4 months p.i. showed normal histology of the CNS, which was totally free of any inflammatory signs.

Luxol fast blue, a special technique for assessing myelin degradation, clearly demonstrates demyelination in areas with perivenular inflammatory infiltrate (Figure 1E). Wide scattered demyelinating plaques (Figure $1 \mathrm{~F}$ ) with characteristic perivenular distribution were present in rats during the paralytic (14 d.p.i.) phase of the disease. Less evident demyelination was also observed in rats during clinical recovery (30 d.p.i.). In addition, the cytoarchitecture of microglia and astrocytes indicated an activated state.

\section{Fibronectin expression in the CNS}

Expression of FN in the CNS of control LEW rats immunized with GPSC plus IFA or saline plus CFA showed a characteristic distribution (Figure 2A), i.e., a fine and delicate mesh on the wall of meningeal blood vessels and in the connective tissue of the choroid plexus, which was almost absent in blood vessels of the nervous tissue. According to the literature (14), FN was not detected in the tissue parenchyma. In contrast, marked immunolabelling was consistently observed in the spinal cord and brainstem vessels (Figure 2B), especially on the luminal surfaces and basal membranes of rats in the pre-clinical phase (7 d.p.i.). Discrete FN labelling restricted to meningeal vessels was observed in the forebrain and midbrain, whereas the brain parenchyma (7 d.p.i) showed no immunolabelling. During the onset of the disease (10 d.p.i.), a marked deposition of FN in the spinal cord and brainstem was observed coincident with appearance of neurological signs (limp tail).

During the paralytic phase (14 d.p.i.), an overdeposition of FN in the spinal cord and brainstem was always associated with perivenular inflammatory cuffs (Figure 2C). Likewise, vessels localized in the same areas but presenting large inflammatory cuffs (Figure 2D) showed only discrete or absent immunolabelling. Dense and rough immunolabelling for FN was detected in the cerebellum, forebrain and midbrain microvessels. Nonetheless, a distinct pattern of FN expression was observed in the thalamus near the third ventricle wall adjacent to few inflammatory cuffs.

After recovery from clinical signs (30 d.p.i.), absent immunolabelling for FN was noticed in microvessels of the spinal cord and brainstem, even in those areas with few inflammatory cuffs. Conversely, FN was detected (Figure 2E) in some vessels of cerebellum, forebrain and midbrain showing small inflammatory cuffs, though it was absent in areas exhibiting large perivenular inflammatory infiltrates (Figure 2F). Interestingly, rats sacrificed four months after sensitization showed a normal characteristic pattern of FN expression in every area of the CNS.

\section{Discussion}

EAE represents an animal model for inflammatory disease of the CNS with damage to the myelin sheaths (demyelination) as a prominent hallmark (8). The severity of disease and distribution of CNS lesions vary depending on the type of antigen used for EAE induction. For instance, distribution of inflammatory lesions in the spinal cord and brainstem rather than in other regions are related to MBP induction, contrasting to MOG- and MAG-specific T-cells that induce numerous lesions in the cerebellar white matter and to S-100ß and GFAP specific Tcells that mediate severe inflammation in the gray matter $(4,5)$.

In the present study we observed a more complex but distinct pattern in the localization of CNS lesions depending on the stage of the disease. The clinical course resembled that obtained following sensitization with whole MBP or MBP fragments (5). During the paralytic phase, affected rats initially 

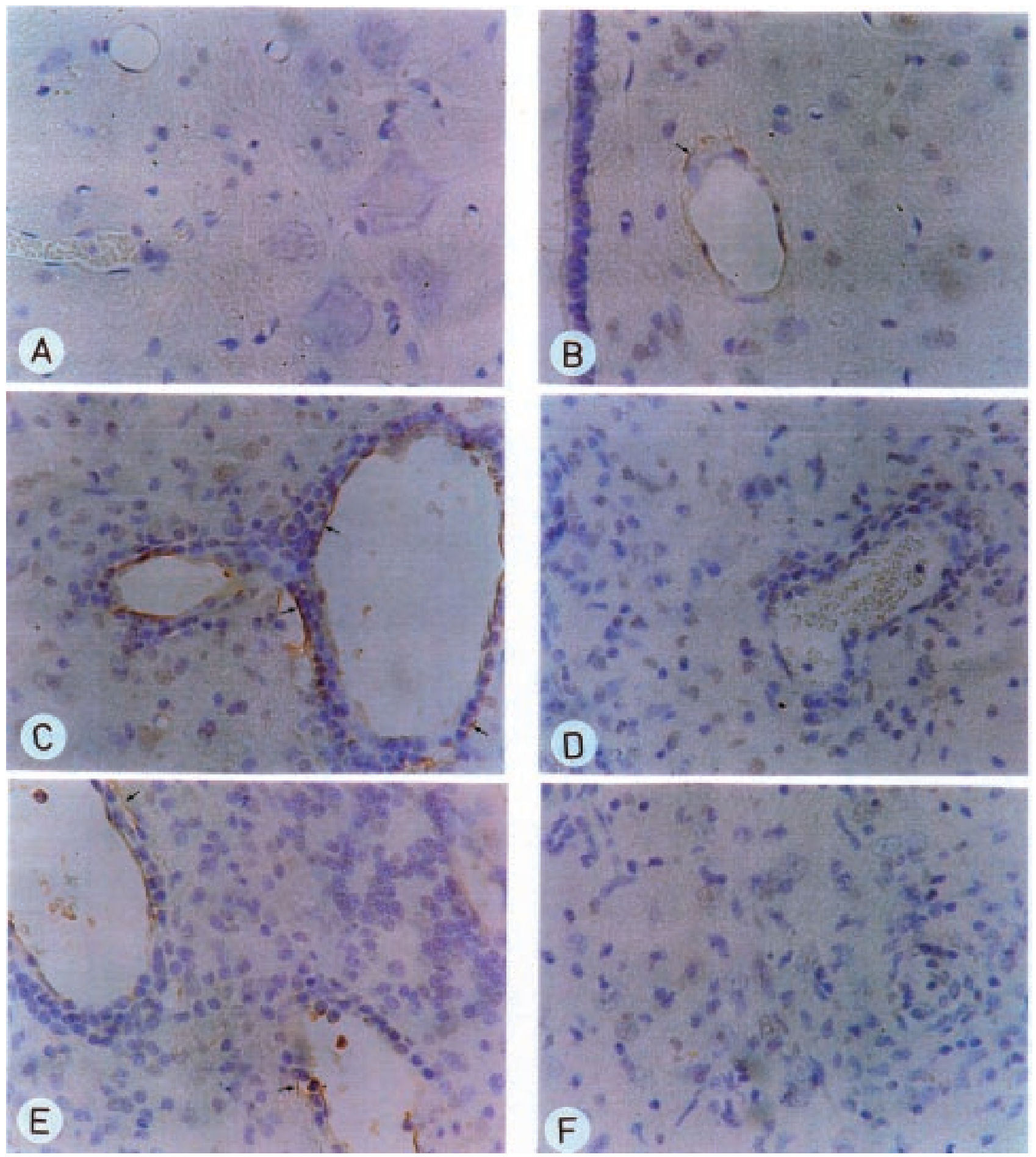

Figure 2 - Photomicrograph of wax-embedded CNS sections from a normal Lewis rat (A) and a rat with EAE (B-F), stained with anti-FN monoclonal antibody. A, Characteristic normal immunolabelling pattern in the cervical spinal cord gray matter segments showing absent FN labelling in the blood vessel walls and nervous parenchyma. B, Lumbosacrococcigeal spinal cord segment from a rat in the pre-clinical phase (7 d.p.i.) showing evident perivenular immunolabelling. C, Intense FN labelling on the luminal surface and basal membrane of a brainstem vessel with a perivascular cuff from rat in the paralytic phase (14 d.p.i.). D, Large perivascular inflammatory infiltrate in a cervical cord segment showing negative FN labelling (14 d.p.i.). E, Cerebellum white matter of a rat sacrificed after clinical recovery (30 d.p.i.) showing intense immunolabelling on the luminal surface and basal membrane of a blood vessel with an inflammatory infiltrate. F, Negative FN immunolabelling of a brain vessel wall and nervous parenchyma with a large inflammatory infiltrate from a rat sacrificed after clinical recovery (30 d.p.i.). Magnification 400X. FN immunolabelling (small arrow). 
showed widespread lesions mainly in the spinal cord and brainstem, but after clinical recovery preferential localization in the cerebellum, midbrain and forebrain was observed. Moreover, inflammatory cuffs were indistinctly located in both gray and white matter. These results may be partly related to the antigenic characteristic of the inoculum (GPSC-CFA homogenate), which contained a mixture of encephalitogenic antigens (MBP, PLP, MOG, MAG).

Among the earliest events in the inflammatory reactions is an increased tendency for circulating leukocytes to adhere to endothelial cell luminal surfaces. Leukocyte migration, antigen recognition, activation and differentiation are events affected by ECM molecules. Moreover, the ability of leukocytes to recognize ECM components may change as they migrate into tissues presumably influenced by signals from the microenvironment (22). A clear modulation of FN expression occurred within the CNS of Lewis rats at distinct phases of active EAE. Initially, there was a dense deposition of FN in the cerebral microvasculature associated with discrete perivenular inflammatory foci, followed by a sharp decrease of FN expression in vessels with numerous inflammatory cuffs. These results suggest an important role for this ECM component guiding the trafficking of leukocytes into the CNS. This assumption is supported by presented evidence that an altered pattern of FN expression during distinct phases of EAE preceded the appearance of inflammatory lesions and demyelination.

Detachment of T cells from the endothelium coincides with down-regulation of integrins (VLA-4, LFA-1) followed by increased binding of transmigrated $\mathrm{T}$ cells to $\mathrm{ECM}$ proteins (type I collagen, type IV collagen and FN) of the interstitial matrix, necessary for establishing residency in the affected area during the course of inflammation (22). The influx of inflammatory cells into the target tissue is associated with remodeling of the ECM (23). In normal biologic processes the maintenance of connective tissue architecture involves controlled breakdown and neosynthesis of ECM elements, requiring timely and limited action of extracellular proteases such as plasminogen activators and matrix metalloproteinases. Leukocyte and endothelial cell-derived metalloproteinases have been implicated in a proteolytic cascade that degrades microvessel wall components, thereby facilitating leukocyte migration into inflammatory sites. The presence of matrilysin near inflammatory lesions of the CNS implicates this protease in the pathogenesis of neuro-inflammatory disease (23, 24). The present work shows that modulation of FN expression in brain vessels always preceded the appearance of inflammatory cells, but faded away after the establishment of the inflammatory cuff and demyelination.

An important aspect to be discussed concerns the putative mechanism(s) involved in the modulation of FN expression during distinct phases of the disease, especially during the paralytic phase. One possibility is that failure to detect perivenular expression of FN near large inflammatory cuffs may be related either to the production of an isoform or altered molecule not recognized by the monoclonal antibody clone MAB1940. Nonetheless, very mild immunolabelling was observed in the same tissue preparation in areas of the CNS without lesions. It is conceivable that the weak or absent FN labelling observed during the paralytic phase may be due in part to the degradation of connective macromolecules by metalloproteinases (e.g., matrilysin) secreted by inflammatory cell cuffs influenced by locally produced cytokines and/or also to intracellular signaling pathways triggered by $\beta 1$ (VLA) and $B 3$ integrin-mediated recogniton of ECM molecules (25).

Taken together, the findings discussed above indicate that increased expression of dense $\mathrm{FN}$ in the CNS microvessels during the pre-clinical phase and the onset of dis- 
ease functions as an attractant directing the trafficking of activated leukocytes to the target tissue. Indeed, such alterations coincided with the appearance of inflammatory cuffs initially in the spinal cord and brainstem, and later ascending into the cerebellum, midbrain and forebrain. It is important to establish, in this model, which of the signals present in the microenvironment of the CNS halt the entrance of activated leukocytes, promote remyelination and render the animal resistant to re-induction of EAE.

\section{Acknowledgments}

We thank Dr. Eliene Carvalho da Fonseca for technical assistance and also the Instituto de Biologia do Exército for providing the guinea pigs.

\section{References}

1. Wekerle H, Kojima K, Lannes-Vieira J, Lassmann H \& Linington C (1994). Animal models. Annals of Neurology, 36: S47S53.

2. Goverman J \& Brabb T (1996). Rodent models of experimental allergic encephalomyelitis applied to the study of multiple sclerosis. Laboratory Animal Science, 46: 482-492.

3. Stepaniak JÁ, Gould KE, Sun D \& Swanborg RH (1995). A comparative study of experimental autoimmune encephalomyelitis in Lewis and DA rats. J ournal of Immunology, 155: 2762-2769.

4. J ohns TG, De Rosbo NK, Menon KK, Abo S, Gonzales MF \& Bernard CA (1995). Myelin oligodendrocyte glycoprotein induces a demyelinating encephalomyelitis resembling multiple sclerosis. J ournal of Immunology, 154: 5536-5541.

5. Berger T, Weerth $\mathrm{S}$, Kojima K, Linington C, Wekerle H \& Lassman H (1997). Experimental autoimmune encephalomyelitis: The antigen specificity of T lymphocytes determines the topography of lesions in the central and peripheral nervous system. Laboratory Investigation, 76: 355364.

6. Sobel RA, Schneeberger EE \& Colvin RB (1988). The immunopathology of acute allergic encephalomyelitis. V. A light microscopic and ultrastructural immunohistochemical analysis of fibronectin and fibrinogen. American J ournal of Pathology, 131: 547-558.

7. Lindsey J W \& Steinman L (1993). Competitive PCR quantification of $C D 4, C D 8$, ICAM-1, VCAM-1, and MHC class II mRNA in the central nervous system during development and resolution of experimental allergic encephalomyelitis. J ournal of Neuroimmunology, 48: 227-234.

8. Olsson T (1995). Critical influences of the cytokine orchestration on the outcome of myelin antigen-specific T-cell autoimmunity in experimental autoimmune encephalomyelitis and multiple sclerosis. Immunological Reviews, 144: 245-268.

9. Campbell J J, Hedrick J, Zlotnik A, Siani MA, Thompson DA \& Butcher EC (1998). Chemokines and the arrest of lymphocytes rolling under flow conditions. Science, 279: 381-384.

10. Steffen BJ, Butcher EC \& Engelhardt B (1994). Evidence for involvement of ICAM-1 and VCAM-1 in lymphocyte interaction with endothelium in experimental autoimmune encephalomyelitis in the central nervous system of the SJ L/J mouse. American J ournal of Pathology, 145: 189-201.

11. J uliano RL \& Haskill S (1993). Minireview: Signal transduction from the extracellular matrix. J ournal of Cell Biology, 120: 577585.

12. Adams J C (1997). Cell adhesion - spreading frontiers, intricate insights. Trends in Cell Biology, 7: 107-110.

13. Yednock TA, Canon C, Fritz LC, Sanchez Madrid F, Steinman L \& Karin N (1992). Prevention of experimental autoimmune encephalomyelitis by antibodies against alpha4 betal integrin. Nature, 356: 63-66.

14. Sobel RA (1998). The extracellular matrix in multiple sclerosis. J ournal of Neuropathology and Experimental Neurology, 57: 205-217.

15. Choi BH (1994). Role of the basement membrane in neurogenesis and repair of injury in the central nervous system. Microscopy Research and Technique, 28: 193-203.

16. Letourneau PC, Condic ML \& Snow DM (1994). Interactions of developing neurons with the extracellular matrix. J ournal of Neuroscience, 14: 915-928.
17. Venstrom KA \& Reichardt LF (1993). Extracellular matrix 2: Role of extracellular matrix molecules and their receptors in the nervous system. FASEB J ournal, 7 : 996-1003.

18. Nagano N, Aoyagi M \& Hirakawa K (1993). Extracellular matrix modulates the proliferation of rat astrocytes in serum-free culture. Glia, 8: 71-76.

19. Garcia-Abreu L, Cavalcante LA, Silva LCF \& Moura-Neto V (1996). The extracellular matrix of the midline and non-midline midbrain glia: correlations with neurite growth-supporting abilities. Brazilian J ournal of Medical and Biological Research, 29: 1179-1187.

20. Paterson PY, Koh C-S \& Kwaan HC (1987). Role of the clotting system in the pathogenesis of neuroimmunology disease. Federation Proceedings, 46: 91-96.

21. Koh C-S, Gausas J \& Paterson PY (1993). Neurovascular permeability and fibrin deposition in the central neuraxis of Lewis rats with cell-transferred experimental allergic encephalomyelitis in relationship to clinical and histopathological features of the disease. J ournal of Neuroimmunology, 47: 141-146.

22. Romanic AM, Graesser D, Baron JL, Visintin I, Janeway J r CA \& Madri J A (1997). T cell adhesion to endothelial cells and extracellular matrix is modulated upon transendothelial cell migration. Laboratory Investigation, 76: 11-23.

23. Kieseier BC, Kiefer R, Clements J M, Miller K, Wells GMA, Schweitzer T, Gearing AJ H \& Hartung H-P (1998). Matrix metalloproteinase-9 and -7 are regulated in experimental autoimmune encephalomyelitis. Brain, 121: 159-166.

24. Clements J M, Cossin J A, Wells GM, Corkill DJ , Helfrich K, Wood LM, Piggot R, Stabler G, Ward GA, Gearing AJ \& Miller 
KM (1997). Matrix metalloproteinase expression during experimental autoimmune encephalomyelitis and effects of a combined matrix metalloproteinase and tumor necrosis factor-alpha inhibitor. J ournal of Neuroimmunology, 74: 85-94.

25. Maeda A \& Sobel RA (1996). Matrix metalloproteinases in the normal human cen- tral nervous system, microglial nodules, and multiple sclerosis lesions. J ournal of Neuropathology and Experimental Neurology, 55: 300-309. 